\title{
E-Healthcare at an Experimental Welfare Techno House in Japan
}

\author{
Toshiyo Tamura ${ }^{*}, 1$, Atsushi Kawarada ${ }^{2}$, Masayuki Nambu ${ }^{3}$, Akira Tsukada ${ }^{4}$, Kazuo Sasaki ${ }^{4}$ and \\ Ken-Ichi Yamakoshi ${ }^{5}$
}

\author{
${ }^{1}$ Chiba University, Japan; ${ }^{2}$ Hiroshima Prefectural University, Japan; ${ }^{3}$ Osaka Electro-Communication University, Ja- \\ pan; ${ }^{4}$ Toyama University, Japan; ${ }^{5}$ Kanazawa University, Japan
}

\begin{abstract}
An automated monitoring system for home health care has been designed for an experimental house in Japan called the Welfare Techno House (WTH). Automated electrocardiogram (ECG) measurements can be taken while in bed, in the bathtub, and on the toilet, without the subject's awareness, and without using body surface electrodes. In order to evaluate this automated health monitoring system, overnight measurements were performed to monitor health status during the daily lives of both young and elderly subjects.
\end{abstract}

Keywords: Smart house, automatic health care system.

\section{INTRODUCTION}

Given the increase in the population of the elderly in our society, we seek to promote a new method of disease prevention using information and communication technology. There are several "smart houses" worldwide that use applied information technology to measure daily health activity at home (for example, Aware House [1]). Epidemiological research is needed to assist in the prevention and identification of diseases such as diabetes and hypertension. Such research requires long-term non-invasive monitoring [2]. Our proposal is to use a smart house that non-invasively monitors physiological parameters. The ability to monitor physiological and vital signs without attaching sensors and transducers to the body is preferable. Thus fully-automated measurements are needed in order to acquire data in a non-invasive manner, and avoid procedures for the subjects. An experimental project on non-invasiveness and the automatic monitoring of a patient's daily physiological status during bathing, excretion, and sleep was conducted at the Welfare Techno House (WTH) in Takaoka, Toyama, Japan.

\section{METHODS}

\subsection{Welfare Techno House}

Because of increasing numbers of elderly in our society, the Japanese government seeks new projects that support and promote the health of the elderly. In 1995, the Japanese Agency of Industrial Science and Technology, under the auspices of the Ministry of International Trade and Industry (MITI), and the New Energy and Technology Development Organizations (NEDO) collaborated to support the construction of 16 demonstration and research houses across Japan, known as Welfare Techno Houses (WTH). The concept of these experimental houses is to promote independence for elderly and disabled persons, and to improve their quality of life. The houses provide an opportunity for clients and caregivers to explore issues concerning accessible design, and to

*Address correspondence to this author at the Chiba University, Japan;

E-mail: tamurat@faculty.chiba-u.jp participate in trials that enable them to meet their own specific needs. The houses are therefore used for testing and exhibition of new products and design concepts. Elderly and disabled people may stay in the houses for several days in order to try out the facilities. In addition, manufacturers are able to test their equipment, although no accreditation is given based on this use. The Ministry of Health and Welfare (presently the Ministry of Health, Labor, and Welfare) supports the integration of information technology with health care. As a result, we have developed an e-healthcare system for the WTH.

The Takaoka Techno House (Fig. 1) is designed to accommodate two generations of people. The two-story building has an area of $400 \mathrm{~m}^{2}$, more than the average Japanese house, and incorporates a range of features that relate to the approach to the house, orientation and movement within, and building management. These features are integrated to provide flexibility of use, and to meet a variety of individual needs, particularly for the elderly who require care. The floor has a cushioning effect and under-floor heating. A home network system is installed as part of the smart house control and communication network to provide control of lighting, curtains, and windows. The security system has a video access-phone connected to the front door.

\subsection{Automatic Healthcare system}

Three automated monitoring systems were installed in the WTH. Electrocardiogram (ECG) measurements were monitored in the bed and the bathtub, and body weight and urinary volume were measured on the toilet (Fig. 2). Fig. (3) shows an ECG recording from the bed. ECGs were taken during sleep via conductive textiles in the pillow and in the sheet beneath the legs [3].

ECGs were also taken during bathing [4,5]. Silver/silver chloride electrodes were installed on the inside wall of the bathtub (Fig. 4) to take ECG recordings through the water. The ECG signal amplitude depends on the conductivity of the water. If the water conductivity is high, the water makes a short circuit to the body as a voltage source, and consequently the amplitude is reduced. By contrast, if the water 

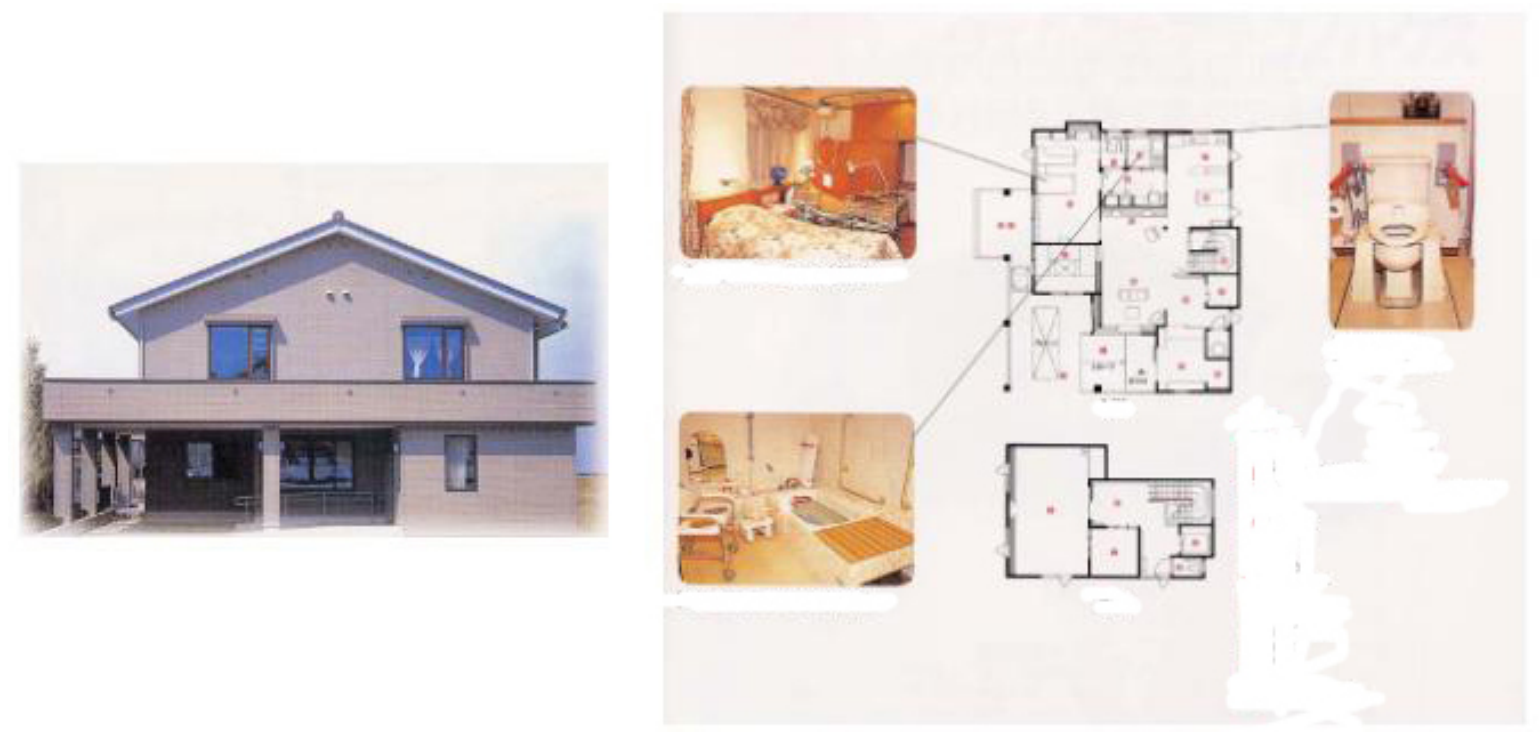

Fig. (1). Overview of Takaoka Welfare Techno House.

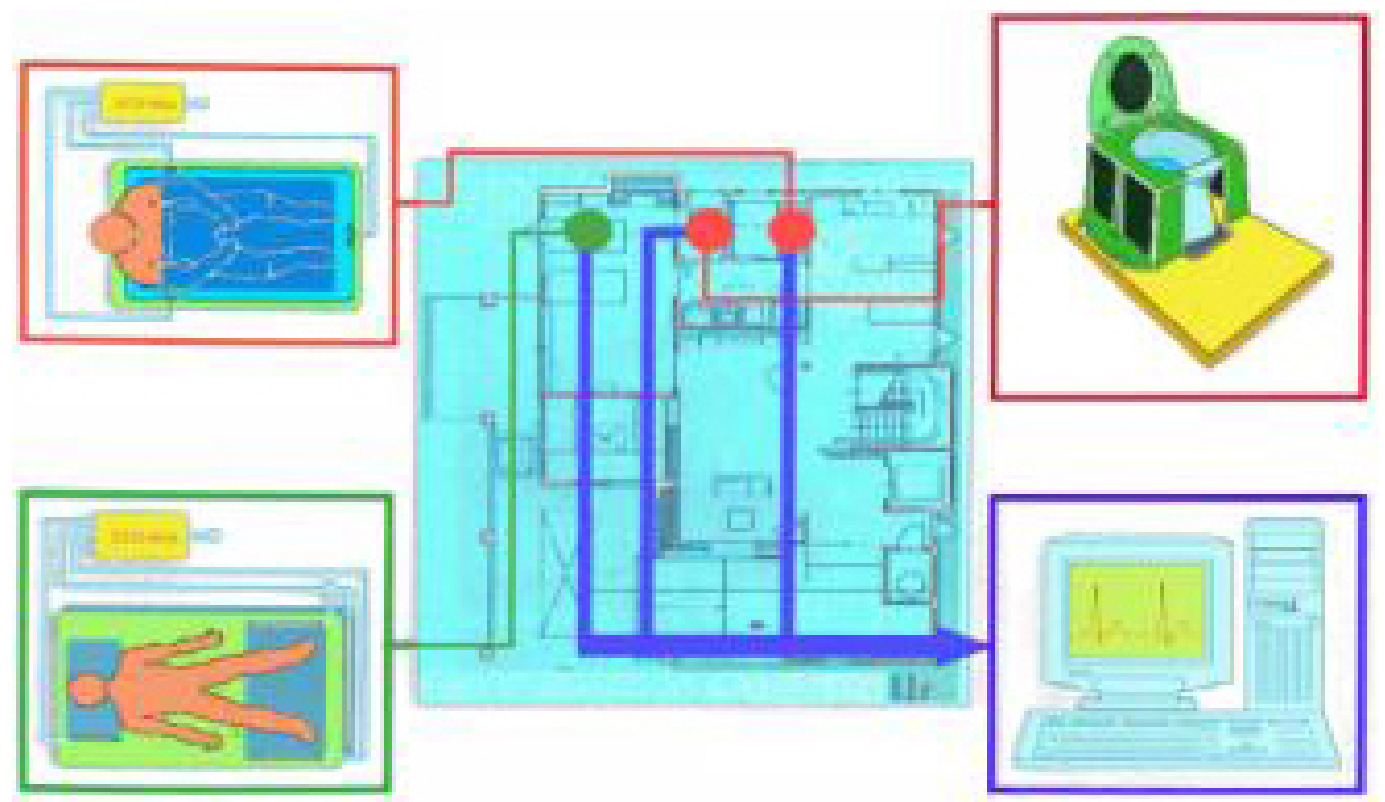

Fig. (2). Floor plan of the monitoring devices.

conductivity is low, signal amplitude remains at levels to similar to those of the skin surface. However, a highimpedance amplifier should be used because of the higher resistance of the water; thus, the recording system becomes more vulnerable to electromagnetic interference. Fortunately, the electric conductivity of ordinary tap water, which is in the order of $10^{-2} \mathrm{~S} \mathrm{~m}^{-1}$, is within an acceptable range of measurement using a conventional amplifier. When subjects entered the tub, large artifacts appeared because of the movement of water near the electrodes and the body surface, but the baseline quickly stabilized, and stable ECGs were then obtained throughout the duration of the stay in the tub.

Body weight and excreta weights were obtained using a weight measuring system that contained four load cells (weight capacity: $200 \mathrm{kgf}$; resolution: $<5$ gf [6]). As shown in Fig. (5), the weight-measuring platform was installed in the floor of the toilet, and the seat was supported so that the weight on the seat was transferred to the load cell. Body weight data can be collected automatically many times each day using this system. In addition, the amounts of urine and feces, and the urine flow rate were monitored, along with habits of urination and evacuation.

\subsection{Data Acquisition}

Data for the physiological parameters were collected in an individual database to determine changes in health status. The computer system consisted of a personal computer (PC) with two A/D converters. Automatic data collection and transfer methods were used, and a personal identification method was required to distinguish among specific subjects.

\subsubsection{Automatic Sampling}


The monitoring system required automatic data downloading to a PC in the home. An automatic data sampling system was developed for each monitor. The appearance of an ECG triggered the start and end of data collection in the bed. In the bathtub, a water level switch triggered the start and end of data collection. On the toilet, fluctuation signals observed when the subjects stood on the load cell or sat on the toilet seat were used as the start and end signals for data collection. Here, the rate of A/D conversion was $256 \mathrm{~Hz}$.

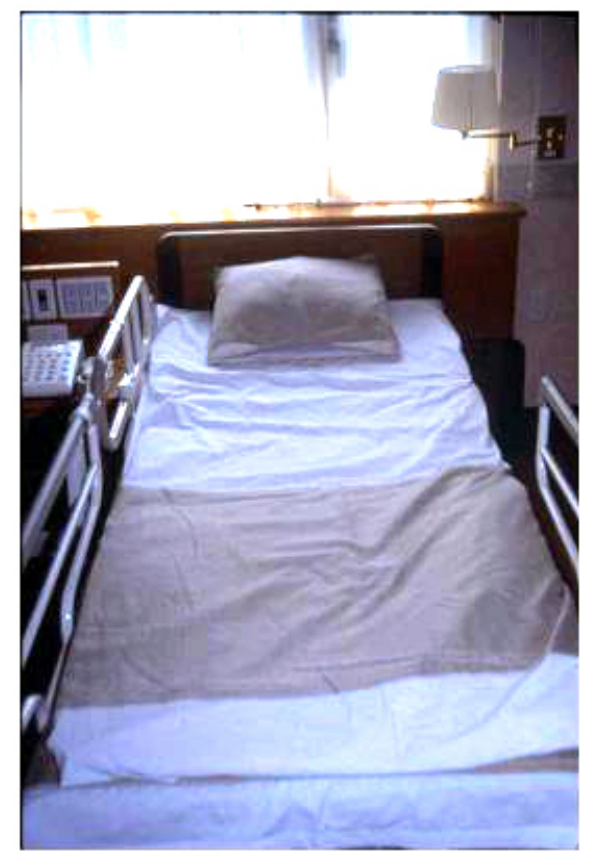

Fig. (3). Bed monitoring system. The electroconductive material was woven into the bed sheet. The pillow and lower part of the sheet shown by the dark colour in the photo are connected to two ECG electrodes.

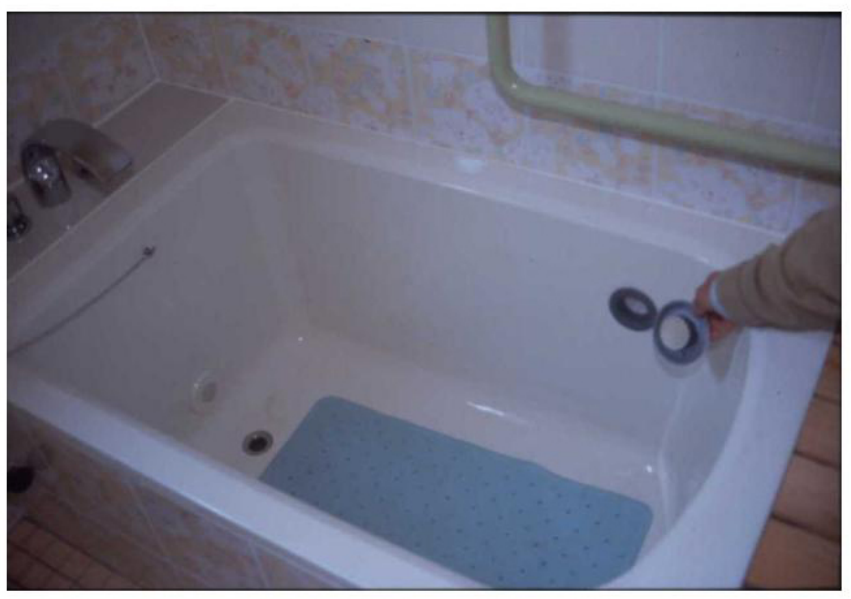

Fig. (4). Bathtub monitoring system.

\subsubsection{Personal Identification}

Personal identification is required because multiple family members may use the monitoring devices. Personal identification of data from the bed was easy because most sub- jects sleep in their own beds. For the bathtub and toilet, the subject was identified before he or she entered the bathroom. However, further research is needed to develop more efficient methods of personal identification.

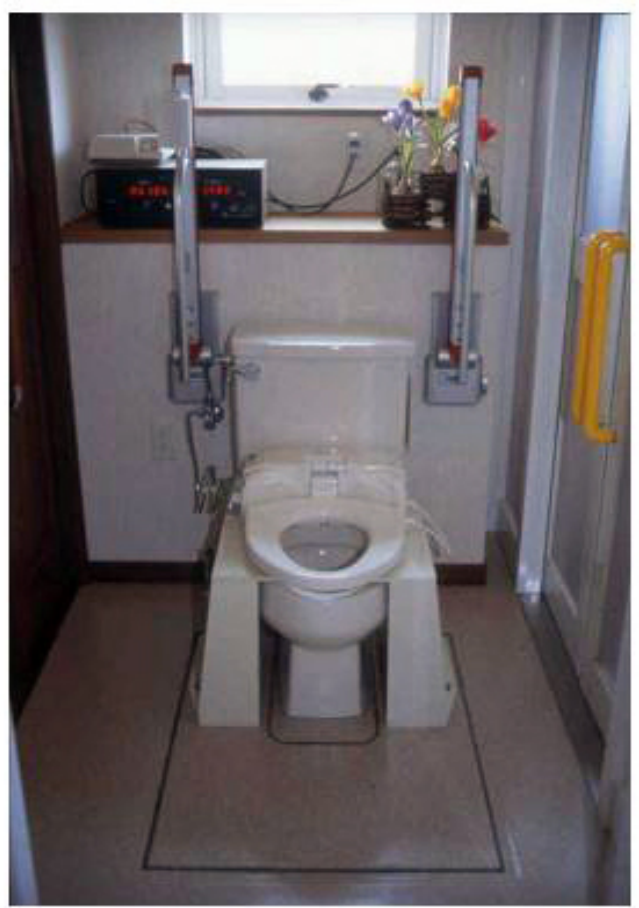

Fig. (5). Toilet weight-monitoring system.

\subsubsection{Data Transfer}

Data from each monitoring system were digitized and stored on the hard disk of a PC in the home. The three monitoring processes must run independently on the PC to obtain simultaneous collection of data. A multitasking operating system performed these tasks simultaneously, without mixing signals or causing interference among tasks. The Linux multitasking operating system allowed three independent monitoring programs to run and store data simultaneously. After each measurement was completed, the data file was created automatically and transmitted to a database in the workstation through a local area network.

The measured data are stored on a PC initially and uploaded for storage on a Linux-based server every day. Each measurement system shares the storage area on the server via network drives using a Windows file system because the data storage area is open to all Windows systems on the local area network using SAMBA server software [7]. Each measurement system copies the data recorded during the day to a shared folder at the scheduled time using batch processing. Simultaneous access cannot occur because the scheduled upload time differs for each measurement system. Data collision cannot occur because the target folder differs for each measurement system, although simultaneous access occurs. In addition, the Linux server can process all access requests simultaneously using its multitasking capacity.

For external access, a Windows NT server is used. Authorised clients can browse the data using the Windows 
Remote Access System (RAS) via a dial-up connection to the Windows NT server.

For both uploading data in the local area network and data browsing from outside the system, all data transfers are executed seamlessly using the Server Message Block (SMB) protocol [7].

\subsection{Trail}

We show a typical example. We added the experimental protocol to the text.

We performed two overnight experiments with seven healthy young male students (average age, $25.3 \pm 5.6$ years) and 11 elderly subjects (three females and nine males average age, $77.5 \pm 3.6$ years). Some of the subjects took part in more than two experiments. Before the experiments, the subjects were explained the experimental protocol and gave written informed consent. The subjects could stay in the experimental house while going about their usual daily activities without any constraints.

\section{RESULTS}

\subsection{Physiological Parameters}

The automatic data collection system functioned without any complications. ECGs were obtained successfully during sleep. Fig. (6) shows a typical example of one night of ECG data taken during sleep. Because the contact between textile electrodes and the skin is not always secure, large artifacts can arise with body movements. If the subject moved, a large artifact occurred. Some signals were affected by respiration (lower right part of Fig. (6)). Thus the ECG should be identified using a high pass filter. Nevertheless, a stable ECG can usually be recorded during sleep more than $70 \%$ of the time [8].
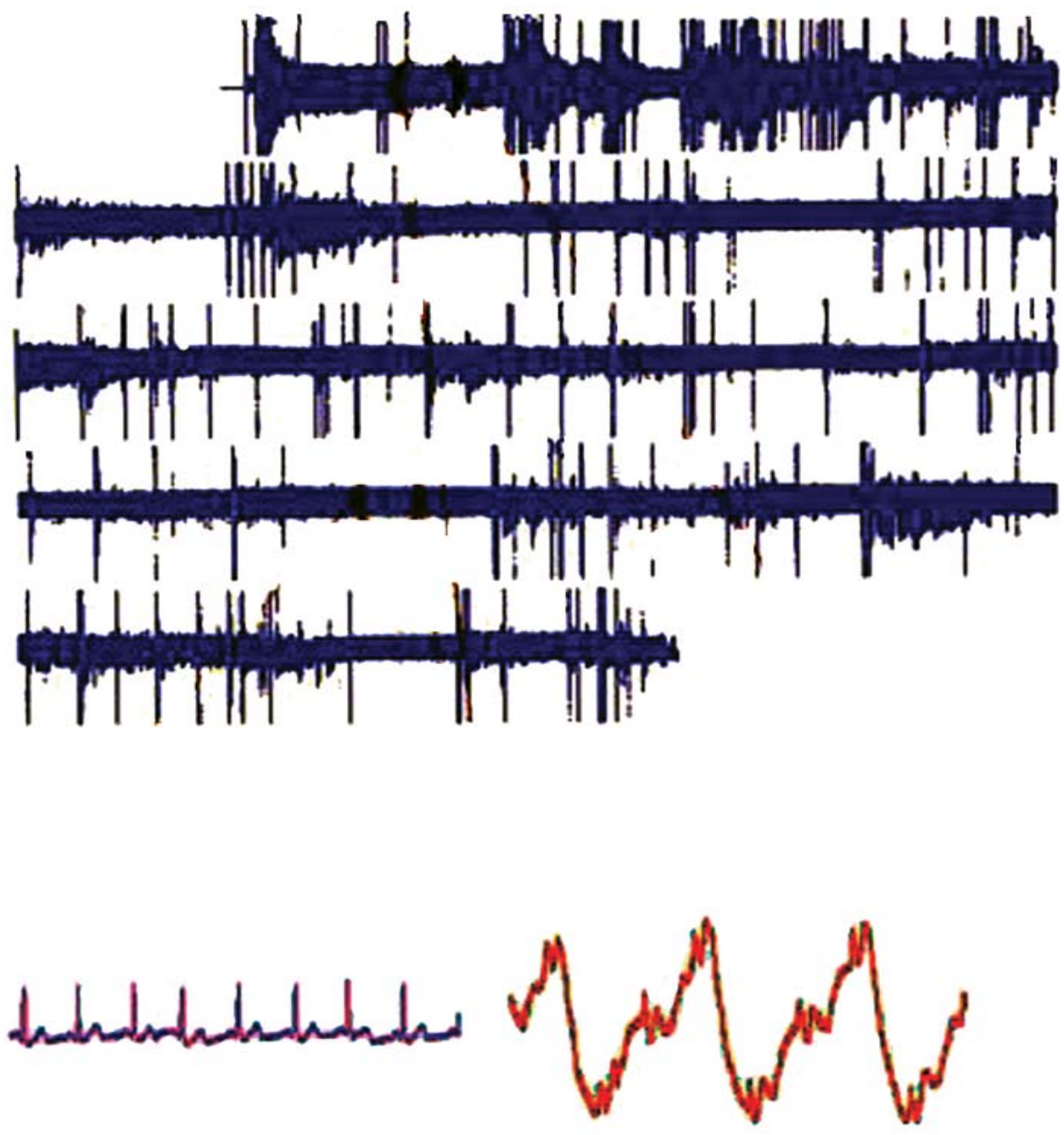

Fig. (6). A typical example of the bed ECG monitor. The bed ECG is monitored during sleep. The large spikelike signals are noise caused by body movement. At rest, the ECG signal can be monitored in the lower-left panel. Most of the signals are influenced by respiration, as shown in the lower-right panel. The ECG signal can be extracted using a high-pass filter. 

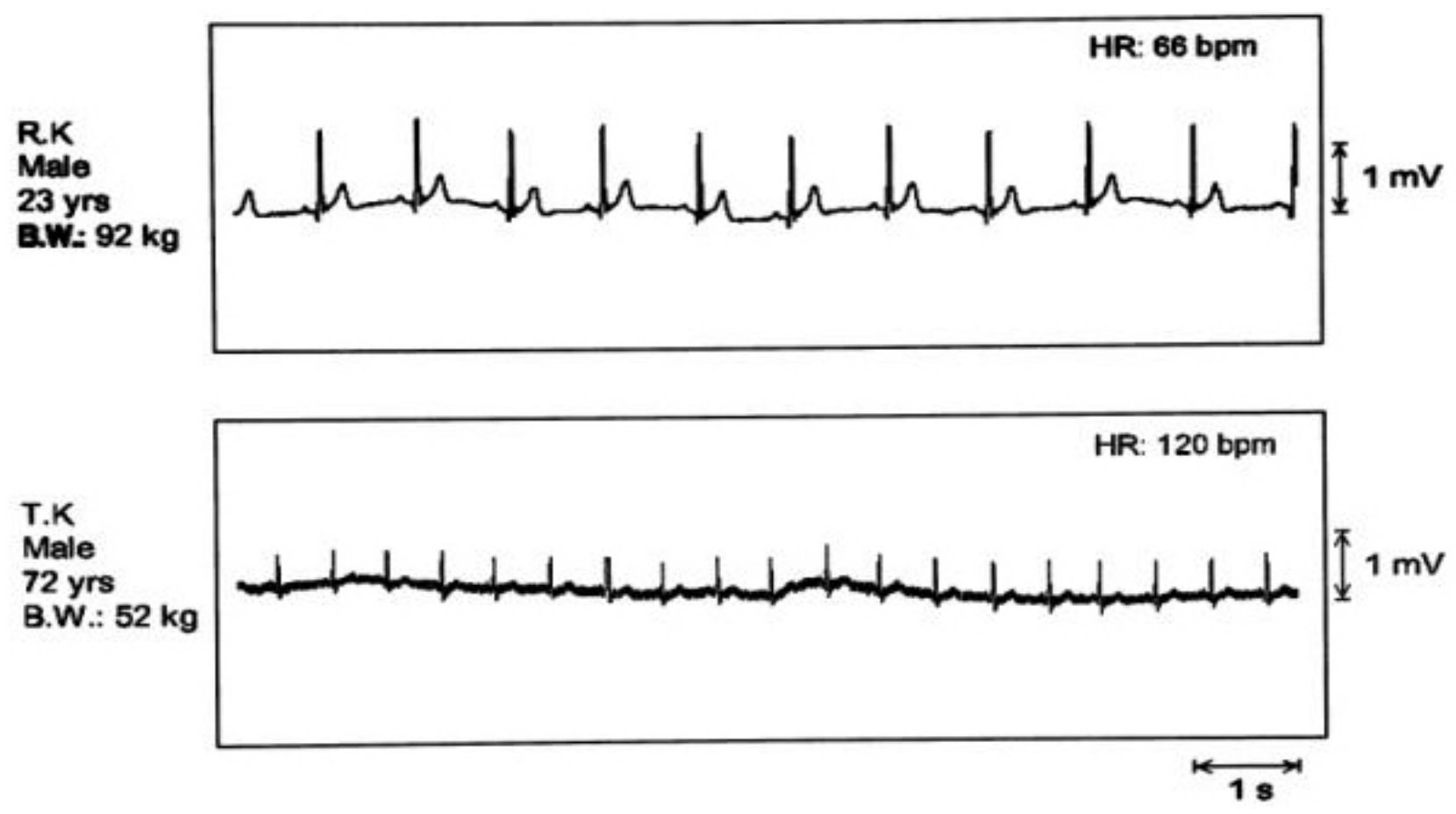

Fig. (7). A typical example of the bathtub ECG monitor. The upper and lower panels show young and elderly subjects, respectively.

ECG signals detected by the bathtub electrodes were recorded clearly in spite of body movements in the bath water. The comparison of ECGs from the bathtub between young and elderly subjects was performed in the same matter as the measurement in the bathtub (Fig. 7). The heart rate of elderly subjects was relatively high compared with that of young subjects. The amplitude of the ECG in elderly subjects was lower than that of young subjects, and the noise level of the ECG of elderly subjects was slightly higher than in young subjects.

Body and excreta weights were successfully measured in a non-invasive and automatic matter. Excreta weight was determined by the difference in body weight between the beginning and end of urination. Fig. (8) shows a comparison of body weight changes during urination between young and elderly subjects. The period of urination of among elderly subjects was longer than among young subjects, and in general the urine weight in elderly subjects was lower than in young ones.

\subsection{Qualitative Analysis-Subject's Impression}

We have provided the questionnaire to the elderly subjects in a separate study. The subjects seem to be satisfied with this system. The advantages that client suggested are automated system. Most elderly subjects are not familiar with handling of the computer. In addition, this system did not need to attach any sensors and transducers to the body. However, the continuous monitoring of physiological parameters is some confusion for the client. Because the device we proposed are cordially clinical evaluation such as surface ECG and blood test. In our ECG monitor, we can consider only heart rate and arrhythmia can be detected.

\section{DISCUSSION}

These preliminary attempts to monitor physiological parameters at the WTH in Takaoka are good examples of preventive health. The system functioned flawlessly during the experiment, and we received continuous monitoring data on each subject. Although more evidence is required, this appears to be a promising model for monitoring health at home. Further investigations are needed to refine the methods for practical use with respect to improved procedures for the automatic start and end of data monitoring and recording, and personal identification.

For ECG monitoring, the devices are only useful for screening purposes. The ECG signal from these devices is not identical to the signal obtained using surface electrodes. Clinically, these data are not reliable because of the attenuation of the signal in the low frequency region.

For urine measurement, prostate enlargement is correlated with urodynamic changes [9]. Therefore, our monitoring system is useful for long-term urodynamic monitoring.

In terms of a business model and integration, the proposed system is very useful for elderly subjects. However, the clients typically only require such monitoring once a week or during an emergency. The questionnaire given to these subjects indicated that continuous monitoring was not required [10]. The proposed system, however, must be refined to better meet their needs.

Issues of privacy and ethics are critical in this system. The elderly were not comfortable with the idea of continuous monitoring, which seemed to rob them of their privacy. The clients wanted to be monitored only when they were sick or during an emergency. We plan to improve and refine our system to make the devices easier to install and handle [11]. 

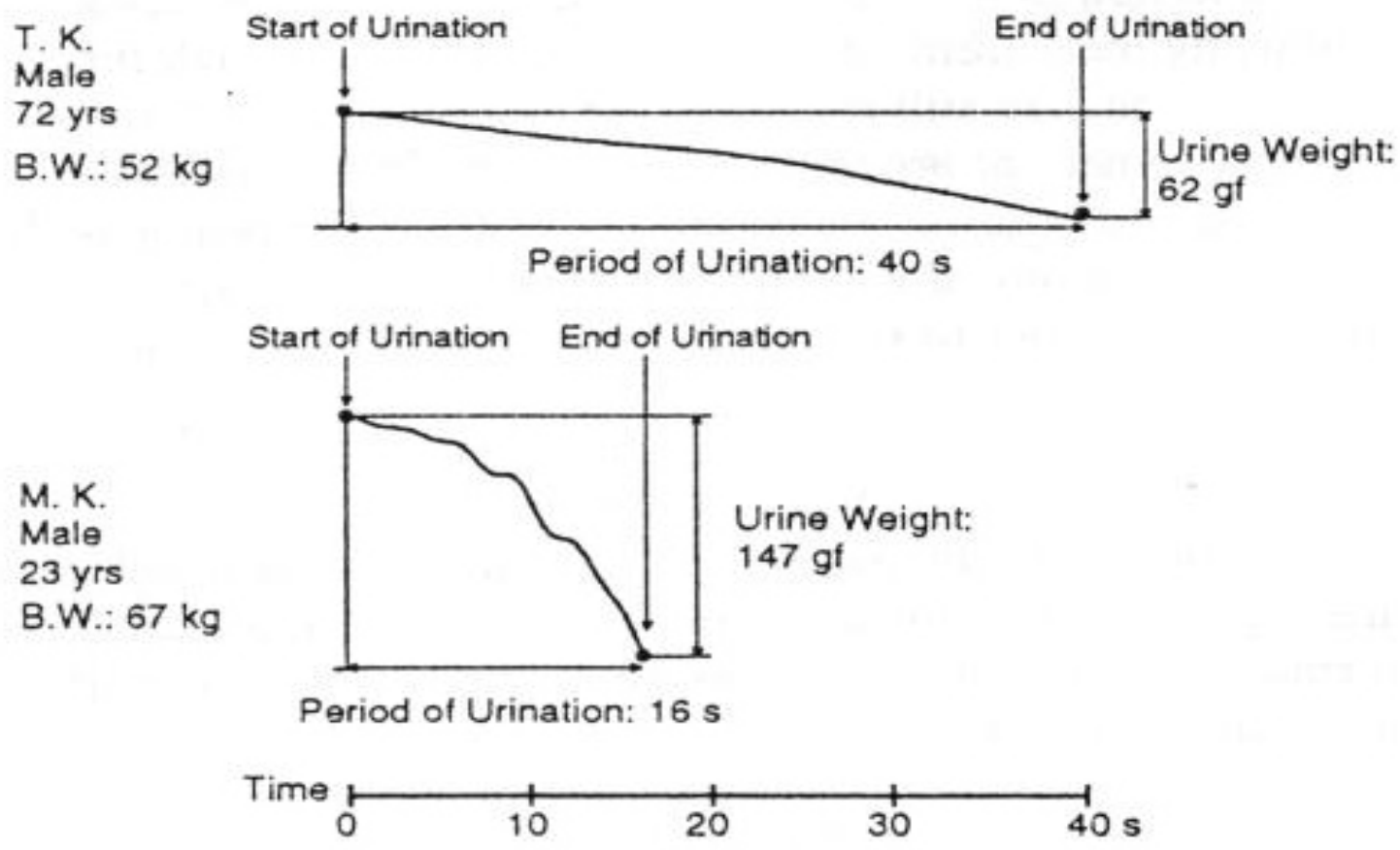

Fig. (8). A typical example of a urine monitor. The upper and lower panels show young and elderly subjects, respectively.

The Techno-House concept is a valuable contribution to the development of accessible domestic dwellings and the integration of these with the information technology infrastructure and daily living products. This technology will facilitate independence and improve the quality of life for elderly and disabled people in Japan. Although few reports have been published on the Japanese small house project, we have proposed innovative new projects and products; however, only one has been commercially implemented (Sekisui, Japan).

The users should be provided with appropriate services when this system is introduced. The data are transferred to the user's hospital and community care centre. The data are stored and analysed based on the results of epidemiological studies. Any difference from normal daily measurements is detected and the client is then notified [12].

Further development needs to evaluate long-term data and predict diseases. It is relatively easy to detect the temporal and sudden abnormal health condition in the period of one or two day. Further in the gradual phenomenon such as hypertension, we proposed the disease prediction each week to find out the regular and irregular daily activity from the obtained health records.

User feedback is very important and we have tried to install these devices in two welfare Techno-Houses. In addition, some companies have built this system commercially. The questionnaire given to the elderly about the usefulness and effectiveness of the automated monitoring system has been discussed elsewhere [10]. Although the measurement concept is rather simple and measurements can be made without the subject being aware, elderly people are not com- fortable with the idea of continuous monitoring. They only want the data transferred when they become ill suddenly or an emergency develops. Normally, the elderly users are informed of their condition once or twice a week. We need to discuss the computer-based system further. The elderly users would prefer an easy-to-install system that could be used only in an emergency or when they become ill [11].

For the security point of view, we treat an almost similar matter as medical records. First of all, the system administrator at this project (University) is responsible for the security of this system or network. Not only it is important to defend the network from outsiders who are trying to gain access to the network, but it is also important to ensure the integrity of the data on the systems within the network.

Traditionally, computer systems have asked for passwords to validate users in the security and privacy. However, for the internet user it is not practical and safety in this method. We used an encryption technology known as public key encryption.

To prevent the disaster, disaster recovery plan is working as data storage of backup data. Backup refers to the process of copying information from disk to a secure storage medium (hard disk ), and backup also refers to the storage medium itself at predetermined time every day.

Reading data for both user and relatives are on the WEB because having the relatively high security protocol

This technology will enable elderly and disabled people in Japan to increase their independence and improve their quality of life. 


\section{CONCLUSIONS}

We developed a fully automatic healthcare system for use in the WTH that effectively collects physiological data. The concept of monitoring physiological parameters are not usual clinical monitor and we proposed ECG monitoring during bathing and sleeping In addition, the body weight, urine volume and speed are monitoring at toilet.

The sensors are installed furniture and sanitary goods and the subject needs to attached the sensor. The heart rate and body weight can be obtained without any special measurement and the subject can received daily physiological parameters without any awareness and discomfort.

It is useful for understanding personal health status and daily activity information without the use of invasive measurements.

\section{ACKNOWLEDGEMENTS}

This work was supported by grants from the Ministry of Health, Labour, and Welfare, Longevity Sciences, and by the CREST project of Chiba University.

\section{REFERENCES}

[1] The Aware House. http: //www.cc.gatech.edu/fce/ahri/ [access on June $\left.10^{\text {th }}, 2007\right]$.

[2] Tamura T, Togawa T, Ogawa M, Yoda M. Fully automated health monitoring system in the home. Med Eng Physics 1998; 20: 573579 .
[3] Ishijima M. Monitoring of electrocardiograms in bed without utilizing body surface electrodes. IEEE Trans Biomed Eng 1993; 40: 593-594.

[4] Tamura T, Yoshimura T, Nakajima K, Togawa T, Miike H. Unconstrained heart rate monitoring during bathing. Biomed Instrum Technol 1997; 31: 391-396.

[5] Ishijima M, Togawa T. Chronodiagnostic acquisition of recovery speed of heart rate under bathing stress. Physiol Meas 1999; 20: 369-375.

[6] Yamakoshi K, Kuroda M, Tanaka S, Yamaguchi I, Kawarada A. A non-conscious and automatic acquisition of body and excreta weight together with ballistocardiogram in a lavatory. Proceeding of the $18^{\text {th }}$ Annual International Conference, IEEE EMBS. 1996; 304.

[7] Samba-opening windows to a wider world. http: //us3.samba.org/ samba/ [access on June 2nd 2007].

[8] Kawarada A, Takagi T, Tsukada A, et al. Evaluation of automated health monitoring system at the "Welfare Techno House". Med Biol Eng Comput 1999; 37(suppl): 112-113.

[9] Van Venrooij GE, Van Melick HH, Eckhardt MD, Boon TA. Correlations of urodynamic changes with changes in symptoms and well-being after transurethral resection of the prostate. J Urol 2002; 168: 605-609.

[10] Watsuji T. The Home Healthcare Project in Japan - the introduction of the system development and the filed test. Proceeding of the Joint Meeting of International Workshop on E-health and 2nd International Conference on Ubiquitous Healthcare 2005; 27-29.

[11] Tsukamoto S, Hoshino H, Tamura T. An easily installable wireless monitoring system for ordinary houses. Proc ICORR 2005 Sherbrooke Canada; 2005.

[12] Nambu M, Nakajima K, Noshiro M, Tamura T. Technological developments in Japan - An algorithm for the automatic detection of health conditions - An image processing technique for diagnosing poor health in the elderly. IEEE Eng Med Biol Mag 2005; 24(4): $38-42$ 